\title{
openheart Diagnostic role of coronary CT angiography in paroxysmal or first diagnosed atrial fibrillation
}

\author{
Dennis Rottländer (D) , ${ }^{1,2}$ Martin Saal, ${ }^{1}$ Hubertus Degen, ${ }^{1}$ Miriel Gödde, ${ }^{1}$
} Marc Horlitz, ${ }^{2,3}$ Michael Haude ${ }^{1}$

To cite: Rottländer D, Saal M, Degen $\mathrm{H}$, et al. Diagnostic role of coronary CT angiography in paroxysmal or first diagnosed atrial fibrillation. Open Heart 2021;8:e001638. doi:10.1136/ openhrt-2021-001638

DR and MS contributed equally.

Received 1 March 2021

Revised 8 April 2021

Accepted 9 April 2021

Check for updates

(c) Author(s) (or their employer(s)) 2021. Re-use permitted under CC BY-NC. No commercial re-use. See rights and permissions. Published by BMJ.

${ }^{1}$ Cardiology, Rheinland Klinikum Neuss GmbH, Neuss, Germany ${ }^{2}$ Cardiology, Faculty of Health, University Witten Herdecke, Witten, Germany ${ }^{3}$ Cardiology, Krankenhaus Porz am Rhein, Köln, Germany

Correspondence to Dr Michael Haude; mhaude@ lukasneuss.de

\section{ABSTRACT}

Objectives The presence of coronary artery disease (CAD) in patients hospitalised with paroxysmal or first diagnosed atrial fibrillation (AF) has major implications for antithrombotic therapy and cardiovascular event rate. Coronary $\mathrm{CT}$ angiography (CCTA) is a feasible tool to identify patients with concealed CAD. We aimed to evaluate the diagnostic role of early CCTA in patients hospitalised with paroxysmal or first diagnosed AF. Methods In a 5-year single-centre retrospective analysis, 566 patients with paroxysmal or first diagnosed AF who underwent CCTA were enrolled to investigate the presence of CAD.

Results In patients with paroxysmal or first diagnosed AF, CCTA revealed CAD (coronary artery stenosis $\geq 50 \%$ ) in $39.2 \%$. Cardiac catheterisation was performed in $31.6 \%$, confirming CAD in $13.1 \%$ of all patients. In $8.0 \%$ percutaneous coronary intervention and in $0.5 \%$ coronary artery bypass grafting was performed. In patients with paroxysmal or first diagnosed AF: (1) angina pectoris per se does not predict CAD; (2) multivariable regression analysis revealed age, male sex and diabetes as risk factors for CAD in AF; (3) Framingham Risk Score for coronary heart disease and $\mathrm{CHA}_{2} \mathrm{DS}_{2}$-VASc-Score were relevant risk scores of $C A D$ and (4) the classification of Coronary Artery Calcium score reference values according to the Multi-Ethnic Study of Atherosclerosis was a predictor of CAD.

Conclusion Patients with paroxysmal or first diagnosed $\mathrm{AF}$ are at risk for CAD, while CCTA is a feasible diagnostic tool for CAD. We recommend to integrate CT calcium scoring and CCTA into the diagnostic workup of patients with new-onset or paroxysmal AF.

\section{INTRODUCTION}

Atrial fibrillation (AF) is associated with an elevated risk of cardiovascular disease such as stroke and myocardial infarction. ${ }^{1}$ Even in patients with idiopathic AF without underlying cardiovascular disease, the cardiovascular event rate is significantly higher than in the general population. ${ }^{2}$ Coronary CT angiography (CCTA) revealed that patients with idiopathic AF more often suffer from concealed and sometimes advanced underlying coronary artery disease (CAD) than

\section{Key questions}

What is already known about this subject?

- Coronary CT angiography (CCTA) is a feasible tool to identify patients with concealed coronary artery disease (CAD).

What does this study add?

- Patients with paroxysmal or first diagnosed atria fibrillation (AF) are at risk of CAD, while angina pectoris per se does not predict CAD in these patients. Male sex and diabetes are risk factors for CAD in AF and Framingham Risk Score for coronary heart disease and $\mathrm{CHA}_{2} \mathrm{DS}_{2}-\mathrm{VASc}$-Score relevant risk scores of CAD. The classification of Coronary Artery Calcium score reference values according to the Multi-Ethnic Study of Atherosclerosis is a predictor of CAD in first diagnosed or paroxysmal AF.

How might this impact on clinical practice?

- CT calcium scoring and CCTA should be considered in the diagnostic workup of patients with new-onset or paroxysmal AF.

controls with sinus rhythm (SR). ${ }^{3} 4$ Early treatment of concealed arteriosclerosis could reduce progression of $\mathrm{CAD}$ and has major influence on the $\mathrm{CHA}_{2} \mathrm{DS}_{2}$-VASc-Score, thus, on potential stroke risk and indication of oral anticoagulation. ${ }^{5}$ CCTA clarifies the diagnosis of CAD, identifies patients requiring percutaneous coronary intervention (PCI) and might reduce the risk of myocardial infarction. The relevance and clinical consequences of CCTA for patients admitted to hospital with first-diagnosed or paroxysmal AF, regardless of their symptoms at the time of admission, remain unclear. The prevalence of CAD might even be higher than in patients with idiopathic AF, since CAD is present in over $20 \%$ of the AF population. ${ }^{6}$ Whether CAD per se predisposes via atrial ischaemia to $\mathrm{AF}$ and how $\mathrm{AF}$ interacts with coronary artery perfusion are uncertain. Recent studies suggest sympathetic activation driven positive 
feedback mechanisms contributing to higher mortality of both diseases. $^{7}$

Several studies of patients admitted to a chest pain unit suggest a beneficial effect of Coronary Artery Calcium (CAC) scoring in risk stratification in AF patients with intermediate pretest likelihood for CAD. ${ }^{89}$ Yet, these studies were conducted in comparably small and preselected study populations at a priori elevated risk for CAD. Recently, Nous et at reported the prognostic value of CCTA in identifying CAD in a small AF patient cohort, emphasising the benefit of CCTA in long term clinical follow-up, but lacking short-term clinical consequences. ${ }^{4}$ Also, none of the published trials used cardiac catheterisation to further evaluate CAD in pathological CCTA.

We aimed to describe the prevalence of CAD diagnosed by CCTA in a representative cohort with paroxysmal or first diagnosed AF and to evaluate the benefit of CCTA derived CAD diagnosis. For the first time, we provide data of invasive coronary angiography and the need of coronary intervention in patients admitted to hospital with first diagnosed or paroxysmal $\mathrm{AF}$ with significant $\mathrm{CAD}$ detected by prior CCTA.

\section{METHODS}

The study was performed in compliance with the Declaration of Helsinki. An individual written consent was obtained by every patient. Patients or the public were not involved in the design, or conduct, or reporting, or dissemination plans of our research.

\section{Study participants}

The study design is summarised in figure 1 . The study cohort comprised 1308 consecutive patients admitted to our hospital with AF between 1 January 2009 and 1 May 2014 who underwent CCTA. Exclusion criteria are listed in figure 1. Patients with persistent, long-term persistent or permanent AF were excluded because of impaired myocardial perfusion, low CCTA image quality due to pulsation artefacts and advanced atrial remodelling leading to atrial cardiomyopathy. ${ }^{10}{ }^{11}$ Patients with spontaneous or iatrogenic conversion into SR were included into the final study cohort. Furthermore, in all patients ECG, echocardiography, CCTA and where available coronary angiography were analysed. Patients were afterwards divided into two groups: (1) patients with $\mathrm{AF}$ and significant $\mathrm{CAD}$ (CAD-AF) and (2) patients with $\mathrm{AF}$ in the absence of significant coronary stenosis (noCAD-AF). Symptoms in AF patients were classified according to the European Heart Rhythm Association (EHRA) classification. $^{12}$

\section{Definition of AF}

$\mathrm{AF}$ was defined as the presence of AF on ECG during the admission to hospital. Electrocardiographic AF was defined as the presence of an irregular rhythm with fibrillatory waves, no defined P-waves and irregular R-wave distances. AF was subclassified into newly diagnosed or paroxysmal AF. ${ }^{1}$

\section{Definition of CAD}

Diagnosis of CAD was established if CCTA or coronary angiography presented a reduction of $50 \%$ or more of the luminal diameter of the coronary arteries. ${ }^{13} \mathrm{~A}$ significant stenosis of the left main artery was considered to be a two vessel disease. Localisation of coronary stenosis was established according to definitions of coronary artery segment as previously described. ${ }^{14}$

\section{Coronary CT angiography}

In all patients calcium scoring including determination of the Agatston score ${ }^{15}$ and calcium mass were performed. Patients were categorised using the CAC score reference values assessed by the Multi-Ethnic Study of Atherosclerosis (MESA) ${ }^{16}$ CT scans were performed using a multislice Brilliance iCT scanner (Phillips Healthcare, Cleveland, Ohio, USA) with a collimation of $256 * 0.6 \mathrm{~mm}$ and rotation time $0.26 \mathrm{~s}$ in accordance with the Society of Cardiovascular CT guidelines. ${ }^{17}$ CCTA was preferably performed in ECG-gated step and shoot technique with a tube current of 200-360 mA at a voltage of $10 \mathrm{kV}$. Native CT for calculation of the calcium mass was performed at $120 \mathrm{kV}$. For automatic bolus tracking, a region of interest was placed in the descending thoracic aorta at a threshold of $150 \mathrm{HU}$. Sublingual application of $0.4 \mathrm{mg}$ gyceroltrinitrate was mandatory before each examination. When necessary, intravenous metoprolol $(5 \mathrm{mg})$ was injected to reach heart rates $<65 / \mathrm{min}$. Steady injection of $70 \mathrm{~mL}$ contrast agent (Imeron 350) with a flow of $5.7 \mathrm{~mL} / \mathrm{s}$ was followed by flushing with $50 \mathrm{~mL}$ saline. The majority of CCTAs was performed by prospective ECG gating at $78 \%$ RR-interval with a slice thickness of $0.5 \mathrm{~mm}$ and a reconstruction interval of $0.3 \mathrm{~mm}$. CCTAs were analysed by two investigators with long-term experience on the field of cardiac CT evaluation (one radiologist and one cardiologist), which were both blinded to the medical data. Coronary arteries were evaluated according to the segment classification of the American Heart Association. ${ }^{14}$

\section{Left heart catheterisation}

Left heart catheterisation was performed as previously described and left ventricular ejection fraction was estimated via angiocardiography. ${ }^{18}$ Coronary arteriography was carried out according to the technique of Judkins using $5 \mathrm{~F}$ catheters. ${ }^{19}$

\section{Statistical analysis}

Statistical analysis was performed using PASW statistics V.18 software (SPSS). All variables were tested for normal distribution with the Kolmogorov-Smirnov test. In the case of normal distribution the results are given as mean $\pm \mathrm{SE}$ of mean, otherwise as median and $95 \%$ CI. Differences between groups and subgroups were evaluated by $\chi^{2}$ test for discrete variables and Student's-t test for continuous variables. For ordinal data Mann-Whitney-U test was used. All clinical covariates showing a univariate relation with the presence of $\mathrm{CAD}$ were included in a logistic regression model and $95 \%$ CIs were calculated. 


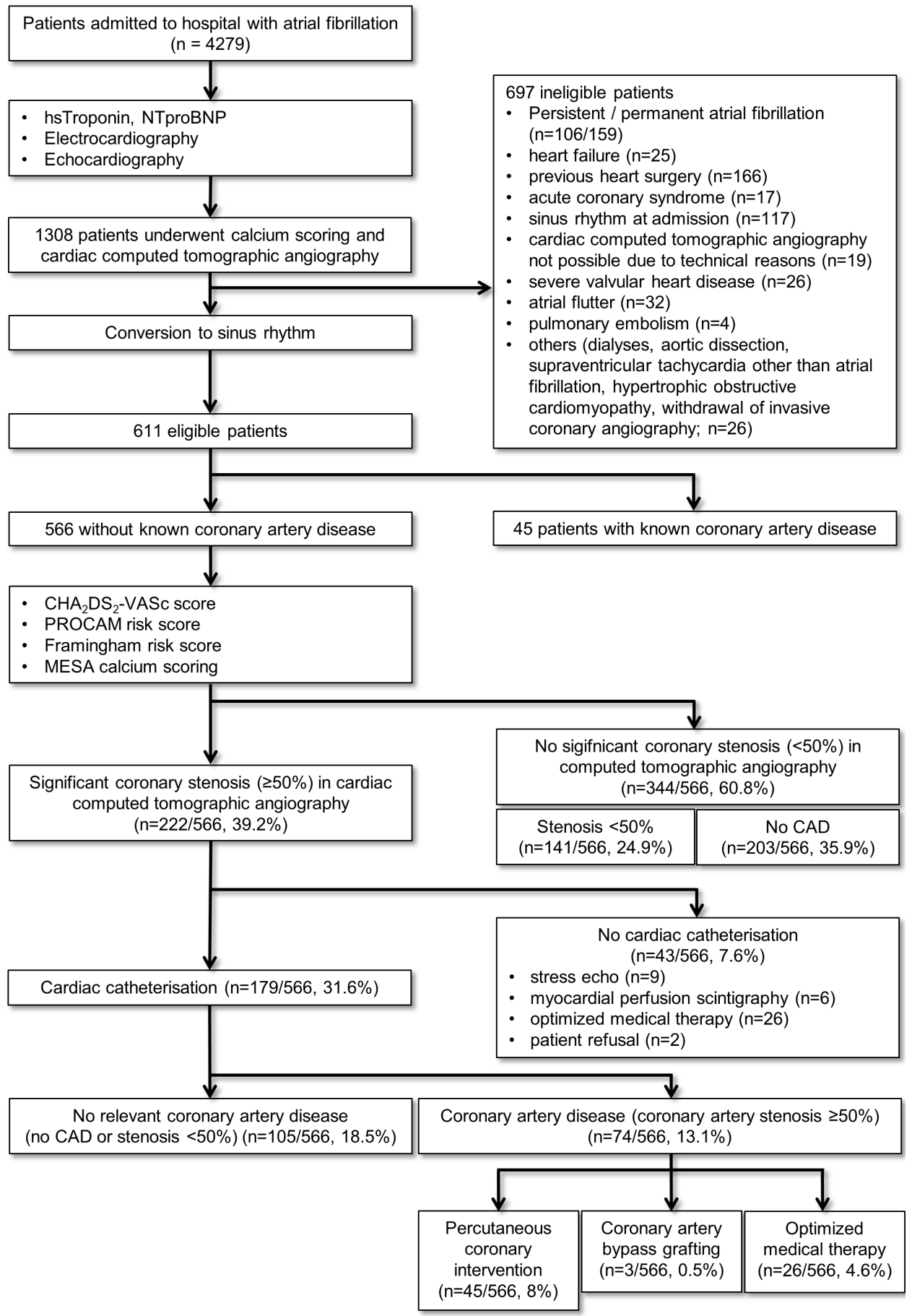

Figure 1 Study design CCTA-AF trial. AF, atrial fibrillation; CCTA, coronary CT angiography; CAD, coronary artery disease; MESA, Multi-Ethnic Study of Atherosclerosis; PROCAM, Prospective Cardiovascular Munster (study); NTproBNP, N-terminal pro Brain natriuretic peptide.

Calibration of the model was checked by the HosmerLemeshow statistic. Statistical significance was assumed at $\mathrm{p}<0.05$.

\section{RESULTS}

\section{Baseline characteristics}

A total of 566 patients with paroxysmal or first diagnosed AF were identified to meet inclusion criteria in this retrospective study (figure 1). Forty-five patients were excluded due to a known history of CAD. In the study population, $27.4 \%$ had paroxysmal AF, whereas $72.6 \%$ presented with first diagnosed AF. The demographic variables of the study population are shown in table 1 .

A total of $48.6 \%$ of the patients were male. Mean age accounts for $67.8 \pm 0.5$ years. Dyspnoea at admission was found in $29.3 \%$ and angina pectoris in 26.3\%. Most of the patients at admission were categorised in EHRA class II (EHRA I 18.2\%, EHRA II 56.4\%, EHRA III 23.5\%, EHRA 
Table 1 Baseline characteristics

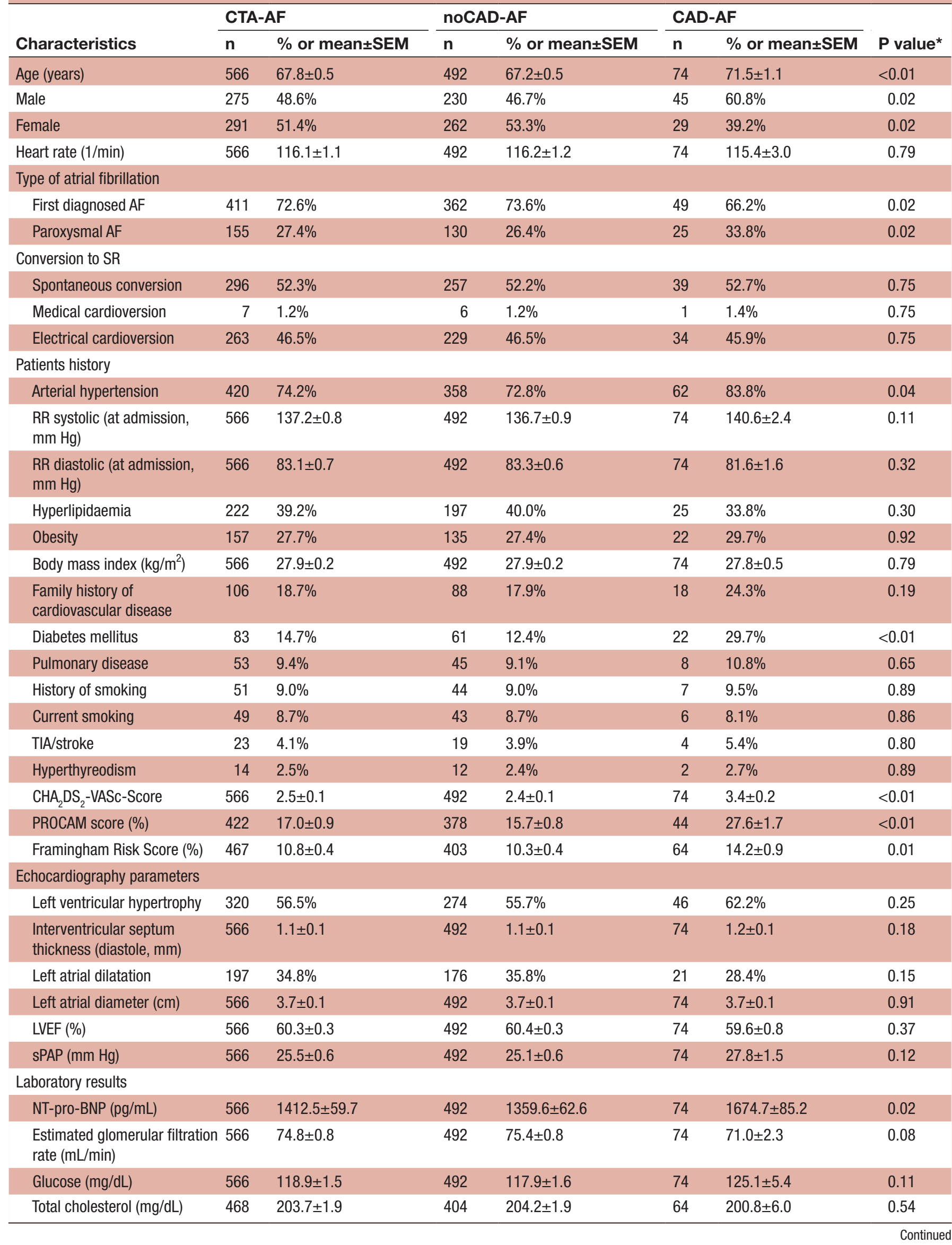


Coronary artery disease

\begin{tabular}{|c|c|c|c|c|c|c|c|}
\hline \multirow[b]{2}{*}{ Characteristics } & \multicolumn{2}{|c|}{ CTA-AF } & \multicolumn{2}{|c|}{ noCAD-AF } & \multicolumn{2}{|c|}{ CAD-AF } & \multirow[b]{2}{*}{ P value* } \\
\hline & $\mathbf{n}$ & $\%$ or mean \pm SEM & $\mathbf{n}$ & $\%$ or mean \pm SEM & $\mathbf{n}$ & $\%$ or mean \pm SEM & \\
\hline $\begin{array}{l}\text { High-density lipoprotein } \\
\text { cholesterol (mg/dL) }\end{array}$ & 468 & $56.5 \pm 0.8$ & 404 & $56.8 \pm 0.9$ & 64 & $54.2 \pm 2.4$ & 0.27 \\
\hline $\begin{array}{l}\text { Low-density lipoprotein } \\
\text { cholesterol (mg/dL) }\end{array}$ & 468 & $123.9 \pm 1.7$ & 404 & $124.1 \pm 1.8$ & 64 & $123.2 \pm 5.3$ & 0.87 \\
\hline \multicolumn{8}{|l|}{ Medication } \\
\hline ACE-inhibitor/AT-1 antagonist & 408 & $72.1 \%$ & 340 & $60.1 \%$ & 68 & $91.9 \%$ & $<0.01$ \\
\hline Diuretics & 253 & $44.7 \%$ & 211 & $37.3 \%$ & 42 & $56.8 \%$ & 0.03 \\
\hline Acetylsalicylic acid & 251 & $44.3 \%$ & 220 & $44.7 \%$ & 31 & $41.9 \%$ & 0.24 \\
\hline Statins & 197 & $34.8 \%$ & 168 & $34.1 \%$ & 29 & $39.2 \%$ & 0.51 \\
\hline Thyroxine & 129 & $22.8 \%$ & 115 & $23.4 \%$ & 14 & $18.9 \%$ & 0.40 \\
\hline $\begin{array}{l}\text { Non-cardioselective } \\
\text { calciumantagonist }\end{array}$ & 101 & $17.8 \%$ & 83 & $16.9 \%$ & 18 & $24.3 \%$ & 0.12 \\
\hline Cumarine & 89 & $15.7 \%$ & 78 & $15.9 \%$ & 11 & $14.9 \%$ & 0.82 \\
\hline Oral antidiabetics & 51 & $9.0 \%$ & 41 & $8.3 \%$ & 10 & $13.5 \%$ & 0.15 \\
\hline DOAC & 34 & $6.0 \%$ & 27 & $5.5 \%$ & 7 & $9.5 \%$ & 0.31 \\
\hline Antiobstructive agents & 32 & $5.7 \%$ & 29 & $5.9 \%$ & 3 & $4.1 \%$ & 0.52 \\
\hline Clopidogrel & 16 & $2.8 \%$ & 13 & $2.6 \%$ & 3 & $4.1 \%$ & 0.32 \\
\hline Digitoxin & 13 & $2.3 \%$ & 12 & $2.4 \%$ & 1 & $1.4 \%$ & 0.56 \\
\hline Class-I antiarrhythmics & 12 & $2.1 \%$ & 12 & $2.4 \%$ & 0 & $0.0 \%$ & 0.18 \\
\hline Insulin & 11 & $1.9 \%$ & 8 & $1.6 \%$ & 3 & $4.1 \%$ & 0.16 \\
\hline Dronedarone & 10 & $1.8 \%$ & 10 & $2.0 \%$ & 0 & $0.0 \%$ & 0.22 \\
\hline $\begin{array}{l}\text { Cardioselective } \\
\text { calciumantagonist }\end{array}$ & 3 & $0.5 \%$ & 2 & $0.4 \%$ & 1 & $1.4 \%$ & 0.50 \\
\hline Amiodarone & 1 & $0.2 \%$ & 1 & $0.2 \%$ & 0 & $0.0 \%$ & 0.70 \\
\hline Betablocker & 433 & $76.5 \%$ & 374 & $76.0 \%$ & 59 & $79.7 \%$ & 0.48 \\
\hline Dyspnoea & 166 & $29.3 \%$ & 139 & $28.3 \%$ & 27 & $36.5 \%$ & 0.15 \\
\hline Stable Angina & 149 & $26.3 \%$ & 131 & $26.6 \%$ & 18 & $24.4 \%$ & 0.42 \\
\hline EHRA I & 103 & $18.2 \%$ & 89 & $18.1 \%$ & 14 & $18.9 \%$ & 0.53 \\
\hline EHRA II & 319 & $56.4 \%$ & 282 & $57.3 \%$ & 37 & $50.0 \%$ & 0.53 \\
\hline EHRA III & 133 & $23.5 \%$ & 111 & $22.6 \%$ & 22 & $29.7 \%$ & 0.53 \\
\hline EHRA IV & 11 & $1.9 \%$ & 10 & $2.0 \%$ & 1 & $1.4 \%$ & 0.53 \\
\hline
\end{tabular}

*noCAD-AF vs CAD-AF.

$\mathrm{ACE}$, angiotensin converting enzyme; AF, atrial fibrillation; AT-1 antagonist, angiotensin-recepector-1 antagonist; CAD, coronary artery disease; CTA, cardiac CT angiography; DOAC, direct oral anticoagulants; EHRA, European Heart Rhythm Association; LVEF, left ventricular ejection fraction; NT-pro-BNP, n-terminal pro brain natriuretic peptide; PROCAM, Prospective Cardiovascular Munster (study); RR, blood pressure; SEM, SE of mean; SPAP, systolic pulmonary artery pressure; SR, sinus rhythm.

IV 1.9\%). Transthoracic echocardiography revealed preserved ejection fraction $(60.3 \% \pm 0.3 \%)$ and normal systolic pulmonary artery pressure (sPAP, $25.5 \mathrm{~mm} \mathrm{Hg}$ \pm 0.6 ). Left ventricular hypertrophy was documented in $56.5 \%$ of the patients, while the left atrium was dilated in $34.8 \%$. However, N-terminal pro nrain natriuretic peptide (NT-pro-BNP) at admission was significantly elevated (1412.5 pg/mL \pm 59.7$)$. Spontaneous conversion into SR was observed in $52.3 \%$, while in $1.2 \%$ medical cardioversion and in $46.5 \%$ electrical cardioversion was conducted. $\mathrm{CHA}_{2} \mathrm{DS}_{2}-\mathrm{VASc}-\mathrm{Score}$ was determined, in most cases indicating long-term oral anticoagulation
(2.5 \pm 0.1 ). Prospective Cardiovascular Munster (PROCAM Risk Score $)(17 \% \pm 0.9 \% ; \mathrm{n}=422)$ and Framingham Risk Score $(10.8 \% \pm 0.4 \% ; \mathrm{n}=467)$ were obtained where applicable. ${ }^{2021}$

\section{Coronary CT angiography}

Mean Agatston score and mean calcium mass were elevated in patients with $\mathrm{AF}$ (120.91 $\pm 8.7,27.61 \pm 2.6$; figure 2A). A negative calcium score was found in $35.9 \%$ of the study population (figure 2B). In patients with paroxysmal or first diagnosed AF CAD ( $\geq 50 \%$ stenosis) was found in $39.2 \%$ using CCTA (figure $2 \mathrm{C}$ ). The mean 

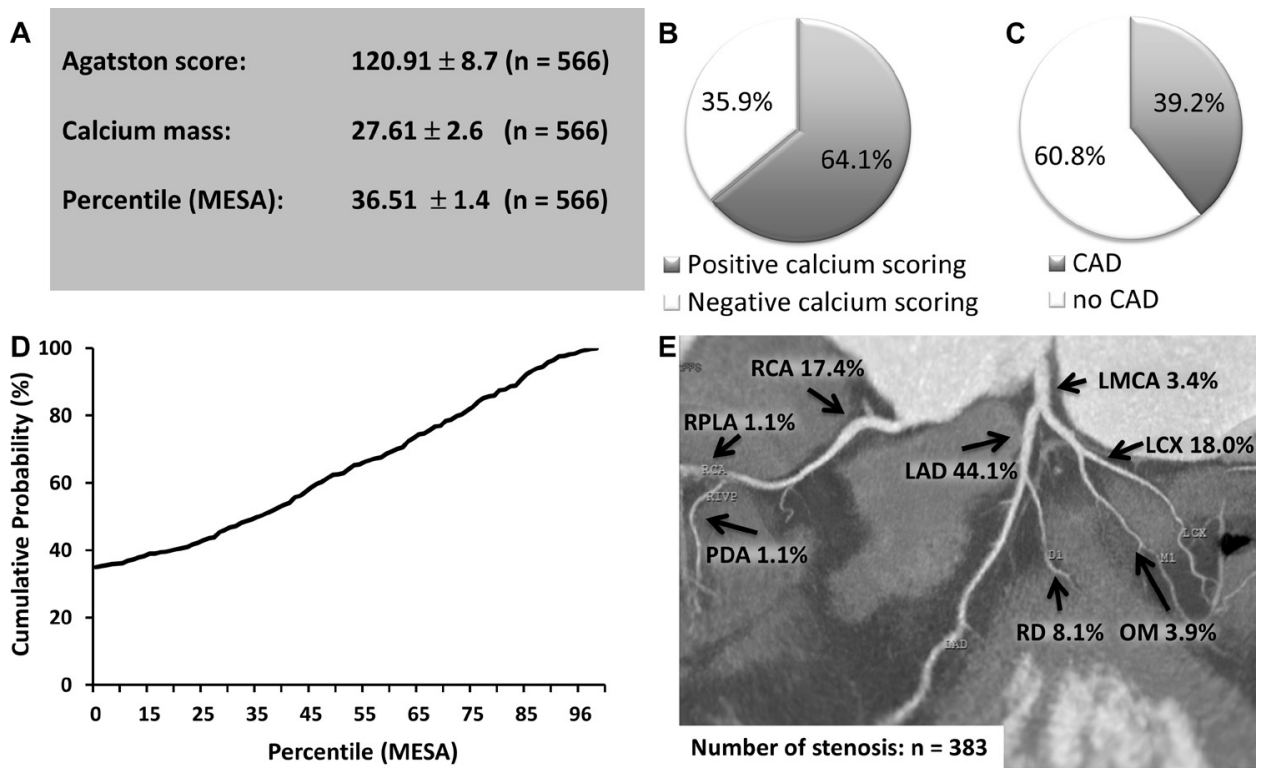

Figure 2 Coronary CT angiography (CCTA) in first diagnosed or paroxysmal AF. (A) Mean results \pm SEM of the calcium scoring including Agatston Score, calcium mass and the Percentile Multi-Ethnic Study of Atherosclerosis (MESA). (B) Percentage of patients with positive and negative calcium scoring. (C) Percentage of CAD positive patients ( $\geq 50 \%$ stenosis) using CCTA. (D) Cumulative distribution function of the percentile MESA score. (E) Localisation of coronary artery stenosis using CCTA in patients with AF. AF, atrial fibrillation; CAD, coronary artery disease; LAD, left artery descending; LCX, left circumflex artery; LMCA, left main coronary artery; OM, Obtuse Marginal Branch; PDA, posterior descending artery; RCA, right coronary artery; $\mathrm{RD}$, diagonal branch; RPLA, right posterolateral artery.

percentile MESA showed intermediate risk for significant $\mathrm{CAD}(36.51 \pm 1.4$, figure $2 \mathrm{~A}, \mathrm{D})$ in patients with $\mathrm{AF}$. In CCTA, coronary stenosis was located in $44.1 \%$ in the left artery descending (LAD), while left circumflex artery (LCX) and right coronary artery (RCA) were affected by significant coronary stenosis in $18.0 \%$ and $17.4 \%$ of the study population, respectively (figure $2 \mathrm{E}$ ).

\section{Cardiac catheterisation}

To verify the results of CCTA, cardiac catheterisation was performed in patients with significant coronary stenosis ( $\geq 50 \%$ stenosis) documented by CCTA. Due to the results of CCTA, cardiac catheterisation was performed in 179 patients $(31.6 \%)$. Left ventricular angiocardiography revealed normal ejection fraction (LVEF, 74.44 \pm 0.7 , figure $3 \mathrm{~A}$ ) and elevated left ventricular enddiastolic pressure (12.93 \pm 0.4 , figure $3 A)$. Significant CAD in the coronary angiogram was observed in $13.1 \%$ of all patients (figure 3B), resulting in PCI in $8.0 \%$ and in coronary artery bypass grafting $(\mathrm{CABG})($ ) in $0.5 \%$ (figure $3 \mathrm{C}$ ). LAD was the predominantly affected segment $(44.3 \%$, figure 3D). Target vessels for PCI were the LAD (40\%), followed by RCA (20\%), LCX (15.5\%) and OM (11.1\%). Notably, in patients with negative calcium scoring (Agatston score 0 ) only one patient needed PCI due to a softplaque $(0.5 \%)$ indicating these patients to be at very low risk of significant CAD.

\section{Characteristics of patients with significant CAD in AF}

In 74 patients significant $\mathrm{CAD}$ was documented using coronary angiography (AF-CAD). This subgroup was compared with patients without significant $\mathrm{CAD}$
(AF-noCAD) in order to evaluate parameters or risk factors for CAD. Demographic variables of both groups are shown in table 1. Univariate analysis revealed age, male sex, arterial hypertension and diabetes mellitus to be significant risk factors for $\mathrm{CAD}$ in $\mathrm{AF}$ (AF-CAD: $71.5 \pm 1.1$ years, $60.8 \%, 83.8 \%, 29.7 \%$ vs AF-noCAD: $67.2 \pm 0.5,46.7 \%, 72.8 \%, 12.4 \%, \mathrm{p}<0.05)$. Furthermore, all evaluated risk scores $\left(\mathrm{CHA}_{2} \mathrm{DS}_{2}\right.$-VASc-Score, Framingham Risk Score, PROCAM Risk Score) were markedly increased in the AF-CAD group (AF-CAD: $3.4 \pm 0.2$, $14.2 \pm 0.9, \quad 27.6 \pm 1.7$ vs AF-noCAD: 2.4 $\pm 0.1, \quad 10.3 \pm 0.4$, $15.7 \pm 0.8, \mathrm{p}<0.05)$. No significant difference in symptoms at admission (angina pectoris or dyspnoea) between both groups could be observed (AF-CAD: 24.4\%, 36.5\% vs AF-noCAD: $26.6 \%, 28.3 \%$, p >0.05). Therefore, angina pectoris is no predictor for significant $\mathrm{CAD}$ in patients admitted to hospital with an episode of first diagnosed or paroxysmal AF. NT-pro-BNP was significantly increased in AF-CAD (AF-CAD: $1763.6 \pm 184$ vs AF-noCAD: $1359.6 \pm 62.6$, $\mathrm{p}<0.05)$.

EHRA classification showed no significant difference between both subgroups (table 1). Calcium scoring was higher in patients with significant CAD (Agatston score: AF-noCAD 96.5 \pm 8.3 vs AF-CAD 283 \pm 8.7 ; calcium mass: AF-noCAD 20.6 \pm 1.7 vs AF-CAD 74.4 \pm 14.9 , p $<0.05$, figure $4 \mathrm{~A})$. The mean percentile $\mathrm{CAC}$ was increased in patients with significant CAD (AF-noCAD 33.1 \pm 1.5 vs AF-CAD 59.4 $\pm 3.3, \mathrm{p}<0.05$, figure $4 \mathrm{~A})$.

In variables showing a univariate relation with $\mathrm{CAD}$, a multivariable regression analysis was performed (table 2). 
A

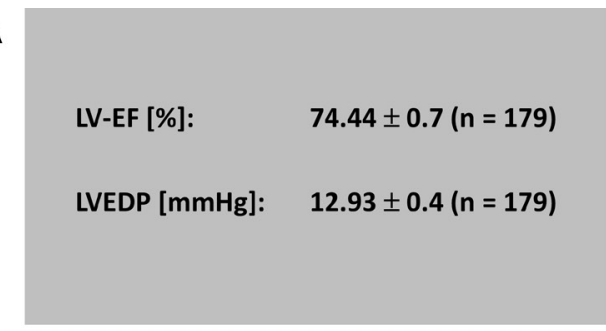

B

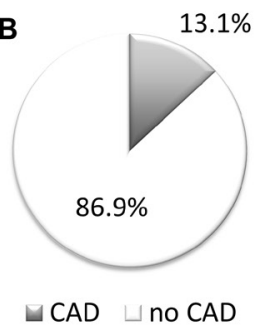

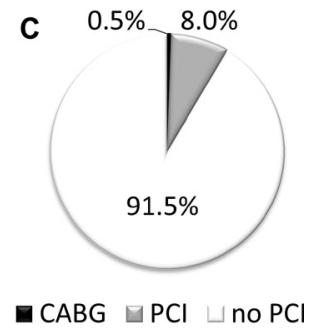

D
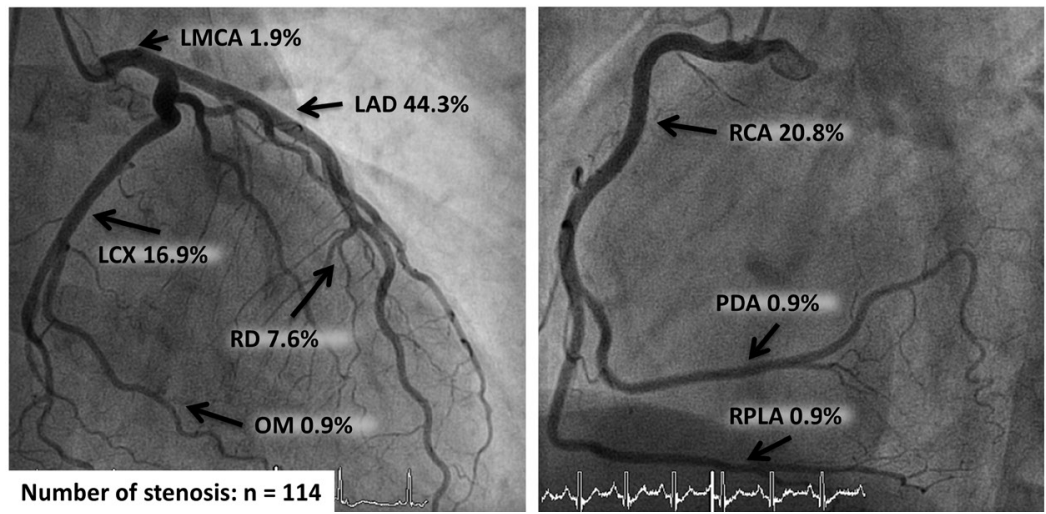

Figure 3 Coronary angiography in patients with AF and pathological CCTA. (A) Angiocardiography results (mean \pm SEM). (B) Percentage of CAD positive patients ( $\geq 50 \%$ stenosis) using coronary angiography. (C) Percentage of patients who underwent $\mathrm{PCl}$ or $\mathrm{CABG}$. (D) Localisation of coronary artery stenosis using coronary angiography. AF, atrial fibrillation; CAD, coronary artery disease; LAD, left artery descending; LCX, left circumflex artery; LMCA, left main coronary artery; LV-EF, left ventricular ejection fractio; LVEDP, left ventricular enddiastolic pressure; OM, obtuse marginal branch; PDA, posterior descending artery; $\mathrm{RCA}$, right coronary artery; RD, diagonal Branch; RPLA, right posterolateral artery.

\section{DISCUSSION}

In this retrospective analysis, we investigated the clinical relevance of early coronary calcium scoring and CCTA in patients with first diagnosed or paroxysmal AF. CCTA showed CAD in $39.2 \%$ of patients with AF. Subclinical CAD is known to be significantly more frequent in patients with idiopathic AF compared with SR controls (49\% vs $34 \%$ ), while obstructive $\mathrm{CAD}$ was found in $4 \%$ of patients with idiopathic $\mathrm{AF}^{3}$ Furthermore, $40 \%$ of patients with paroxysmal and persisting AF showed obstructive CAD in CCTA, ${ }^{22}$ which matches our results. This strengthens the hypothesis that $\mathrm{AF}$ could be a marker of CAD. For the first time, we report data for calcium scoring and CCTA in AF followed by cardiac catheterisation in obstructive CAD. Invasive coronary angiography revealed obstructive CAD in $13.1 \%$ of the patients. Furthermore, $8.0 \%$ of the patients required PCI and $0.5 \%$ CABG. This demonstrates that patients with first diagnosed or paroxysmal $\mathrm{AF}$ are at risk of obstructive CAD.

Nous et al reported $26 \%$ of patients with obstructive CAD in CCTA and $29 \%$ of the patients with CAD underwent coronary revascularisation. This is moderately higher than in our study, where $20.3 \%$ underwent coronary intervention (45 of 222 patients with CAD in CCTA underwent $\mathrm{PCI}$ and two received $\mathrm{CABG}$ ). This might be due to slightly different baseline characteristics, as our cohort presents less well factors for CAD, in particular regarding current or history of smoking. Yet, the need for PCI in our study was far higher than in studies investigating significant CAD by CCTA in a cohort of 73 patients admitted to a chest pain unit. ${ }^{8}$ In this cohort, $7 \%$ needed coronary intervention (two patients with PCI and three with CABG). This comparably low necessity of intervention emphasises our findings that there is only a weak association between AF patients admitted to hospital due to chest pain and relevant CAD. This applies especially for patients presenting with atypical chest pain, which was one inclusion-criteria in the aforementioned studies.

Angina pectoris during $\mathrm{AF}$ was not a significant predictor of $\mathrm{CAD}$ in our cohort, which has major implications for the clinical management of patients with first diagnosed or paroxysmal AF. This suggests that patients with $\mathrm{AF}$ and concomitant angina pectoris need not universally be classified as acute coronary syndrome, unless further diagnostics confirm this diagnosis. In the long term, identification of patients at high risk for CAD might be mandatory to reduce cardiovascular events. Therefore, early detection of underlying $\mathrm{CAD}$ in $\mathrm{AF}$ using CCTA will lead to adequate clinical treatment of risk factors and $\mathrm{CAD}$. For example, certain antiarrhythmics must be considered carefully and lipids need to be monitored and treated more intensively. ${ }^{23}{ }^{24}$ Newly diagnosed CAD might also trigger the need for oral anticoagulation due to elevated $\mathrm{CHA}_{2} \mathrm{DS}_{2}$-VASc-Score. ${ }^{1}$ Furthermore, we showed calcium scoring to be a useful diagnostic tool. Diabetes mellitus is a major risk factor for $\mathrm{CAD}$ also in $\mathrm{AF}$. In the Framingham Risk Score, but not in the PROCAMRisk-Score, Diabetes mellitus is included in risk assessment indicating that Framingham Risk Score is more suitable for risk assessment in patients with $\mathrm{AF}^{20}{ }^{21} 25$ The non-inclusion 


$\begin{array}{lcc}\text { A } & \text { AF-noCAD } & \text { AF-CAD } \\ \text { Agatston score: } & 96.5 \pm 8.3(n=492) & 283.3 \pm 8.7(n=74) * \\ \text { Calcium mass: } & 20.6 \pm 1.7(n=492) & 74.4 \pm 14.9(n=74) * \\ \text { Percentile (MESA): } & 33.1 \pm 1.5(n=492) & 59.4 \pm 3.3(n=74) *\end{array}$

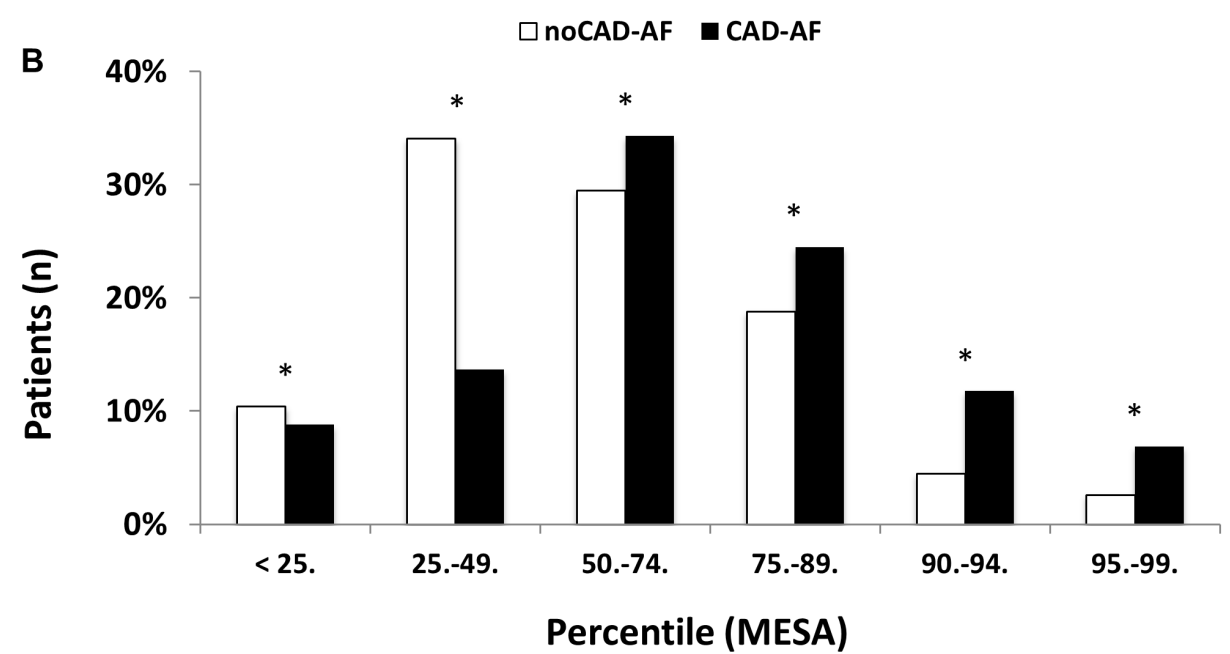

Figure 4 Comparison of patients with early stage AF in absence (AF-noCAD) or presence of significant CAD (AF-CAD). (A) Comparison of calcium scoring including the Agatston Score, calcium mass and percentile acquired by the Coronary Artery Calcium Score reference values assessed by the Multi-Ethnic Study of Atherosclerosis (MESA) (mean \pm SEM). (B) Patients categorised into groups using the Percentile MESA. ${ }^{*} P<0.05$. AF, atrial fibrillation; $C A D$, coronary artery disease;

Table 2 Regression analysis of risk factors, risk scores and calcium scoring

\begin{tabular}{|c|c|c|c|}
\hline & OR & $95 \% \mathrm{Cl}$ & $P$ value \\
\hline \multicolumn{4}{|l|}{ Risk factors } \\
\hline Age (years) & 1.053 & 1.023 to 1.084 & $<0.01$ \\
\hline Male sex (\%) & 1.042 & 1.246 to 1.714 & $<0.01$ \\
\hline Diabetes mellitus (\%) & 2.768 & 1.536 to 4.990 & $<0.01$ \\
\hline \multicolumn{4}{|l|}{ Risk scores } \\
\hline $\mathrm{CHA}_{2} \mathrm{DS}_{2}$-VASc-Sscore & 1.398 & 1.060 to 1.844 & 0.018 \\
\hline Framingham Risk Score & 1.851 & 1.060 to 3.244 & 0.047 \\
\hline \multicolumn{4}{|l|}{ Calcium scoring } \\
\hline Calcium Mass & 1.497 & 1.098 to 1.214 & 0.016 \\
\hline Percentile MESA & 1.016 & 1.106 to 1.627 & $<0.01$ \\
\hline
\end{tabular}

Multivariable regression analysis of risk factors, risk scores and calcium scoring parameters related to the presence of significant coronary artery disease in paroxysmal or first diagnosed AF. Age, male sex and diabetes were significant risk factors for $C A D$ in first diagnosed or paroxysmal AF. Furthermore, CHA2DS2VASc -Score and Framingham Risk Score showed a significant association with CAD. PROCAM risk score failed to reach significance. The analysis of calcium scoring revealed calcium mass and mean percentile CAC to be predictive for significant CAD in AF.

$\mathrm{AF}$, atrial fibrillation; $\mathrm{CAC}$, coronary artery calcium; $\mathrm{CAD}$, coronary artery disease; MESA, Multi-Ethnic Study of Atherosclerosis. of diabetes mellitus in the PROCAM-Risk-Score may be the reason, why it failed to reach significance in our study.

The incidence of coronary events in AF varies among studies, since the selection criteria of patients or the definition of coronary events are heterogeneous. The RE-LY trial showed an incidence of $0.53 \%$ for myocardial infarction during a 2-year follow-up in the warfarin arm. ${ }^{26}$ The REACH registry, where patients with high-risk profiles were enrolled, reported $1.36 \%$ non-fatal myocardial infarction in $\mathrm{AF}$ patients. ${ }^{27}$ To avoid cardiovascular events in patients with $\mathrm{AF}$, the presence of $\mathrm{CAD}$ should carefully be controlled. The present study investigated the benefit of early CCTA in AF and aimed to identify the patients at high risk for relevant CAD. Early detection and treatment of even subclinical CAD is useful to prevent coronary events and to identify patients for oral anticoagulation regarding the $\mathrm{CHA}_{2} \mathrm{DS}_{2}$-VASc-Score. ${ }^{28}$

In summary, this study demonstrated that patients with paroxysmal or first diagnosed $\mathrm{AF}$ are at risk for $\mathrm{CAD}$. For the first time we evaluated a large cohort of patients with $\mathrm{AF}$ and CCTA which underwent invasive coronary angiography in case of pathological CCTA. Age, male sex and diabetes mellitus are risk factors for $\mathrm{CAD}$ in $\mathrm{AF}$, while Framingham Risk Score and $\mathrm{CHA}_{2} \mathrm{DS}_{2}$-VASc-Score are significant risk scores for $\mathrm{CAD}$ in $\mathrm{AF}$. The classification according to MESA is a valuable CT predictor for $\mathrm{CAD}$ in AF. This 
has major impact on $\mathrm{CHA}_{2} \mathrm{DS}_{2}$-VASc-Score, antithrombotic therapy and probably on the cardiovascular event rate. Overall, CCTA is a feasible diagnostic tool for CAD in patients with first diagnosed or paroxysmal AF. Therefore, we recommend to integrate CT calcium scoring and CCTA into the diagnostic workup of patients with new-onset or paroxysmal AF.

\section{STUDY LIMITATIONS}

This study is of retrospective nature. A prospective randomised trial is needed to verify our results. Regarding prevention of cardiovascular events due to early detection of $\mathrm{CAD}$ in $\mathrm{AF}$, a long-term follow-up would be needed to detect significant differences due to the low short-term incidence of cardiovascular events in these patients.

\section{Twitter Dennis Rottländer @DRottlander}

Contributors DR: conceptualisation; data collection, analysis and interpretation; drafting; final approval. MS: data collection, analysis and interpretation; drafting; final approval. MG: data collection. HD: conceptualisation, critical revision. MH: conceptualisation; critical revision; final approval.

Funding The authors have not declared a specific grant for this research from any funding agency in the public, commercial or not-for-profit sectors.

Competing interests $\mathrm{HD}$ is a consultant for Biotronik and Cardiac Dimensions. MH received institutional grants/research supports from Abbott, Biotronik and receipt of honoraria or consultation fees from Biotronik, Cardiac Dimensions, OrbusNeich and Philips. The other authors have nothing to declare.

Patient consent for publication Not required.

Ethics approval Since we performed a retrospective analysis of our patient database, no ethical approval is required due to local regulations.

Provenance and peer review Not commissioned; externally peer reviewed.

Data availability statement Data are available on reasonable request.

Open access This is an open access article distributed in accordance with the Creative Commons Attribution Non Commercial (CC BY-NC 4.0) license, which permits others to distribute, remix, adapt, build upon this work non-commercially, and license their derivative works on different terms, provided the original work is properly cited, appropriate credit is given, any changes made indicated, and the use is non-commercial. See: http://creativecommons.org/licenses/by-nc/4.0/.

ORCID iD

Dennis Rottländer http://orcid.org/0000-0002-8789-9092

\section{REFERENCES}

1 Hindricks G, Potpara T, Dagres N, et al. 2020 ESC guidelines for the diagnosis and management of atrial fibrillation developed in collaboration with the European association for Cardio-Thoracic surgery (EACTS). Eur Heart J 2021;42:373-498.

2 Potpara TS, Stankovic GR, Beleslin BD, et al. A 12-year followup study of patients with newly diagnosed lone atrial fibrillation: implications of arrhythmia progression on prognosis: the Belgrade atrial fibrillation study. Chest 2012;141:339-47.

3 Weijs B, Pisters R, Haest RJ, et al. Patients originally diagnosed with idiopathic atrial fibrillation more often suffer from insidious coronary artery disease compared to healthy sinus rhythm controls. Heart Rhythm 2012;9:1923-9.

4 Nous FMA, Budde RPJ, van Dijkman ED, et al. Prognostic value of subclinical coronary artery disease in atrial fibrillation patients identified by coronary computed tomography angiography. $A m \mathrm{~J}$ Cardiol 2020;126:16-22.

5 Lip GYH, Nieuwlaat R, Pisters R, et al. Refining clinical risk stratification for predicting stroke and thromboembolism in atrial fibrillation using a novel risk factor-based approach: the Euro heart survey on atrial fibrillation. Chest 2010;137:263-72.

6 Michniewicz E, Mlodawska E, Lopatowska P, et al. Patients with atrial fibrillation and coronary artery disease - Double trouble. Adv Med Sci 2018;63:30-5.
7 Lieder H, Breithardt G, Heusch G. Fatal attraction - A brief pathophysiology of the interaction between atrial fibrillation and myocardial ischemia. Int J Cardiol 2018;254:132-5.

8 Breuckmann F, Olligs J, Hinrichs L, et al. Coronary artery calcium as an independent surrogate marker in the risk assessment of patients with atrial fibrillation and an intermediate pretest likelihood for coronary artery disease admitted to a German chest pain unit. Clin Cardiol 2016;39:157-64.

9 Koopmann M, Hinrichs L, Olligs J, et al. Cardiac computed tomography in patients with symptomatic new-onset atrial fibrillation, rule-out acute coronary syndrome, but with intermediate pretest probability for coronary artery disease admitted to a chest pain unit. Eur J Med Res 2018;23:6.

10 Range FT, Schäfers M, Acil T, et al. Impaired myocardial perfusion and perfusion reserve associated with increased coronary resistance in persistent idiopathic atrial fibrillation. Eur Heart $J$ 2007;28:2223-30.

11 Goette A, Kalman JM, Aguinaga L, et al. EHRA/HRS/APHRS/ SOLAECE expert consensus on atrial cardiomyopathies: definition, characterization, and clinical implication. Europace 2016;18:1455-90.

12 Kirchhof P, Auricchio A, Bax J, et al. Outcome parameters for trials in atrial fibrillation: recommendations from a consensus conference organized by the German Atrial Fibrillation Competence NETwork and the European Heart Rhythm Association. Europace 2007;9:1006-23.

13 Thygesen K, Alpert JS, Jaffe AS, et al. Fourth universal definition of myocardial infarction (2018). Eur Heart J 2019;40:237-69.

14 Austen WG, Edwards JE, Frye RL, et al. A reporting system on patients evaluated for coronary artery disease. Report of the Ad Hoc Committee for Grading of Coronary Artery Disease, Council on Cardiovascular Surgery, American Heart Association. Circulation 1975;51:5-40.

15 Agatston AS, Janowitz WR, Hildner FJ, et al. Quantification of coronary artery calcium using ultrafast computed tomography. J Am Coll Cardiol 1990;15:827-32.

16 McClelland RL, Chung H, Detrano R, et al. Distribution of coronary artery calcium by race, gender, and age: results from the multi-ethnic study of atherosclerosis (MESA). Circulation 2006;113:30-7.

17 Abbara S, Blanke P, Maroules CD, et al. SCCT guidelines for the performance and acquisition of coronary computed tomographic angiography: a report of the Society of Cardiovascular Computed Tomography Guidelines Committee: endorsed by the North American Society for Cardiovascular Imaging (NASCl). J Cardiovasc Comput Tomogr 2016;10:435-49.

18 Dodge HT, Sandler H, Ballew DW, et al. The use of biplane angiocardigraphy for the measurement of left ventricular volume in man. Am Heart J 1960;60:762-76.

19 Judkins MP. Percutaneous Transfemoral selective coronary arteriography. Radiol Clin North Am 1968:6:467-92.

20 Assmann G, Schulte $\mathrm{H}$, Cullen $\mathrm{P}$, et al. Assessing risk of myocardial infarction and stroke: new data from the prospective cardiovascular Münster (PROCAM) study. Eur J Clin Invest 2007;37:925-32.

21 D'Agostino RB, Vasan RS, Pencina MJ, et al. General cardiovascular risk profile for use in primary care: the Framingham heart study. Circulation 2008;117:743-53.

22 Nucifora G, Schuijf JD, van Werkhoven JM, et al. Relationship between obstructive coronary artery disease and abnormal stress testing in patients with paroxysmal or persistent atrial fibrillation. Int $J$ Cardiovasc Imaging 2011;27:777-85.

23 Greenberg HM, Dwyer EM, Hochman JS, et al. Interaction of ischaemia and encainide/flecainide treatment: a proposed mechanism for the increased mortality in cast I. Br Heart $J$ 1995;74:631-5.

24 Knuuti J, Wijns W, Saraste A, et al. 2019 ESC guidelines for the diagnosis and management of chronic coronary syndromes. Eur Heart J 2020;41:407-77.

25 Kannel WB, Abbott RD, Savage DD, et al. Coronary heart disease and atrial fibrillation: the Framingham study. Am Heart $J$ 1983;106:389-96.

26 Connolly SJ, Ezekowitz MD, Yusuf S, et al. Dabigatran versus warfarin in patients with atrial fibrillation. $N$ Engl J Med Overseas Ed 2009;361:1139-51.

27 Goto S, Bhatt DL, Röther J, et al. Prevalence, clinical profile, and cardiovascular outcomes of atrial fibrillation patients with atherothrombosis. Am Heart J 2008;156:855-63.

28 Miyasaka Y, Barnes ME, Gersh BJ, et al. Coronary ischemic events after first atrial fibrillation: risk and survival. $A m \mathrm{~J}$ Med 2007;120:357-63. 